\title{
Multidisciplinary Nutrition Care: Benefitting Patients with Malnutrition Across Healthcare Sectors
}

\author{
Heather Keller, Susan Slaughter, Leah Gramlich, \\ Ashwini Namasivayam-MacDonald, and Jack J. Bell
}

\begin{abstract}
Geriatric malnutrition prevention, detection, and treatment benefit from a multidisciplinary approach, regardless of the care setting. Nutrition care pathways have been created to support multidisciplinary care for hospitals and for transitions and primary care. Conceptual models for supporting nutrition in long-term care emphasize a multidisciplinary approach.
\end{abstract}

This chapter is a component of Part II: Special Topic in Geriatric Nutrition.

For an explanation of the grouping of chapters in this book, please see Chap. 1: "Overview of Nutrition Care in Geriatrics and Orthogeriatrics."

\section{H. Keller $(\bowtie)$}

Schlegel-University of Waterloo Research Institute for Aging, University of Waterloo,

Waterloo, ON, Canada

e-mail: hkeller@uwaterloo.ca

\section{S. Slaughter}

Faculty of Nursing, University of Alberta, Edmonton, AB, Canada

e-mail: sslaught@ualberta.ca

\section{Gramlich}

Department of Medicine, Faculty of Medicine and Dentistry, University of Alberta,

Edmonton, AB, Canada

e-mail: lg3@ualberta.ca

\begin{abstract}
A. Namasivayam-MacDonald
School of Rehabilitation Science, McMaster University, Hamilton, ON, Canada

e-mail: namasia@mcmaster.ca
\end{abstract}

\section{J. J. Bell}

Allied Health, The Prince Charles Hospital, Chermside, Queensland, Australia

School of Human Movement and Nutrition Sciences, The University of Queensland, St Lucia, QLD, Australia

e-mail: jack.bell@health.qld.gov.au

Ó. G. Geirsdóttir, J. J. Bell (eds.), Interdisciplinary Nutritional Management and 


\section{Keywords}

Acute care $\cdot$ Long-term care $\cdot$ Malnutrition $\cdot$ Multidisciplinary care $\cdot$ Nutrition assessment $\cdot$ Nutrition therapy $\cdot$ Nutritional support

\section{Learning Outcomes}

By the end of this chapter, you will be able to:

- Explain the difference between screening and assessment and how different healthcare professionals can take part in detection of geriatric malnutrition.

- Describe the various roles the multidisciplinary team contributes to the prevention, detection, and treatment of malnutrition.

- Formulate a plan for multidisciplinary geriatric malnutrition care using existing nutrition care pathways and conceptual frameworks designed for specific sectors.

\subsection{Malnutrition, Assessment, and Screening}

Malnutrition is defined as the inadequate intake or use of energy and nutrients resulting in changes in body composition that impact mental and cognitive function and impair clinical outcomes from acute disease [1]. Malnutrition is extremely common in older adults [2, 3]. Prevalence varies based on how malnutrition is assessed [4], as well as disease subpopulations, but is approximated at a third of acute care patients [5] and up to half of residents in long-term care [6]. Prevalence of malnutrition in community living older adults is more elusive [7]. Nutrition risk precedes malnutrition and is an indication that the individual has risk factors that impair food intake or has increased requirements which could lead to malnutrition: prevalence is approximated at one-third of community-living older adults [8]. This prevalence necessitates a multidisciplinary approach, as access to dietitians, whose scope of practice includes malnutrition assessment and treatment, is limited across all sectors.

Nutrition screening is a quick and easy-to-complete process of identifying nutrition risk using a validated screening tool [1]. Malnutrition screening can be completed by any healthcare professional with minimal training. Electronically captured data can also be used to screen for malnutrition, and in some settings, patientadministered tools are available $[9,10]$. As these tools vary, the training requirements will also vary. For example, screening tools that require the measurement of body mass index or calf circumference (e.g., Mini Nutritional Assessment-Short Form [11]) will require more training than tools that are based on questions (e.g., Canadian Nutrition Screening Tool [12]). Screening tools may be specific to the population and sector for which they are created. For example, the Canadian Nutrition Screening Tool [12] is recommended for acute care, and the SCREEN-14 for community-living seniors [13]. Other tools can be used across sectors, such as the MNA-SF [11]. 
A comprehensive nutrition assessment includes anthropometry and body composition measures; nutrition-focused biochemistry; a history of dietary intake and adequacy of this intake; food security; assessment of functional capacity; a physical exam for muscle and fat wasting and physical signs of micronutrient deficiency; clinical history of disease states, medications and surgeries, and substance abuse issues; and social history (e.g., living situation, food-related activities of daily living, such as grocery shopping, cooking, and eating ability) [1]. The clinician completing a nutritional assessment tailors these components dependent on the age, disease, and other contexts of the patient (e.g., living in the community vs. longterm care).

\subsection{Multidisciplinary Roles in Malnutrition Care}

\subsubsection{Dietitian Role}

Dietitians in many countries of the world have been trained to complete a comprehensive, individualized nutrition assessment to detect malnutrition and counsel patients [14] on nutritional means for mitigating malnutrition (e.g., low-dose vitamin pills, oral nutritional supplements (ONS), diet enhancements, diet modifications, etc.), as well as other strategies to support nutritional status (e.g., the use of adapted utensils, grocery delivery, etc.). Dietitians have specialized knowledge on nutrient requirements for age, sex, and disease groups, as well as a comprehensive understanding of the nutritional value of food and supplements [15], and as such can tailor a therapeutic diet to support nutritional status and health goals. As malnutrition assessment and treatment are a core area of practice, dietitians are readily able to detect and treat malnutrition. However, not all dietitians have the specialized knowledge for malnutrition care in geriatrics, especially considering the complexity of comorbid conditions, frailty [16], and dementia [17] in this subgroup. Further, dietitians are a limited resource in all sectors of healthcare [18, 19], and it is not realistic that all at-risk patients will see a dietitian for treatment [20]. The greater healthcare team is needed to support malnutrition care.

\subsubsection{Physician Role}

Another important member of the multidisciplinary team is the physician. Physicians can play a central role in identifying malnutrition. Some physicians are nutrition specialists and have been trained to complete a comprehensive nutritional assessment. However, a majority of physicians, with training, are able to identify risk and diagnose malnutrition using standardized tools such as the subjective global assessment [21]. Where dietitians are involved in assessing patients, the dietitian can communicate the diagnosis of malnutrition to the physician, allowing prioritization of appropriate nutrition care. Physicians are also central to identifying, diagnosing, and treating symptoms which preclude oral intake. In addition, they are well 
positioned to anticipate the patients' trajectory and how medical care and intercurrent illness will impact nutrition care in a given patient. In addition to identifying risk and/or diagnosing malnutrition, physicians are integral to communicating and emphasizing the importance of a malnutrition diagnosis to older patients and how this malnutrition may be linked to their other health conditions or geriatric syndromes, including frailty as discussed in Chap. 7. The physician is well poised to support nutritional treatment by prescribing oral nutritional supplements [22], higher-dose micronutrients, as well medications to mitigate potential causes of poor food intake. A critical function of the physician is working with dietitians and other health professionals to implement complex nutrition care processes, such as artificial feeding or how to address continuing weight loss.

\subsubsection{Role of the Speech-Language Pathologist}

Speech-language pathologists (SLPs) are integral to the multidisciplinary team as some, and specifically those with a geriatric practice, are specialists in swallowing and swallowing impairments (dysphagia). SLPs, however, are not readily available in all care settings, especially in under-resourced areas. Dysphagia is independently associated with malnutrition and dehydration in older adults [23]. Food and fluid intake may be reduced or eliminated for many older adults with both acute and chronic conditions, such as a post-stroke, post brain injury, dementia, Parkinson disease, head and neck cancer, etc., due to risk for aspiration (i.e., food or liquid entering the airway, increasing risk for pneumonia). Food and fluid may also be modified in texture, which is unpalatable for many patients, leading to reduced intake. Finally, modified texture food itself may lead to malnutrition if it is not sufficient in energy and micronutrients [24]. Dysphagia is prevalent among older adults. Similar to malnutrition, prevalence is dependent on the assessment method (bedside or instrumental), patient population, and setting. Poststroke, acute dysphagia is estimated to be present in up to $80 \%$ of patients [25], while dysphagia risk due to a variety of geriatric-related diseases, including dementia, could be as high as $60 \%$ in long-term care [26]. It is also present in up to $29 \%$ of community-dwelling older adults [27]. SLPs have the requisite skills to assess risk of aspiration and the swallowing physiology and function of patients. Working with the team, they recommend and train the team and patient on compensatory strategies (e.g., chin tucks, clearing swallows), provide rehabilitative treatment (e.g., tongue-strengthening exercises and other exercises to improve physiology), and recommend any necessary diet modifications. As dysphagia can resolve or worsen depending on the primary medical diagnosis and other complicating factors, it is ideal that the SLP be involved in the ongoing reassessment of swallowing capacity for patients with dysphagia, to further limit malnutrition and dehydration. Dietitians, physicians, and nurses often include dysphagia risk screening for select geriatric groups (e.g., poststroke, Parkinson disease, dementia) referring those with potential risk to SLP for assessment, when this resource is available. Commonly used screening tools include the Toronto Bedside Swallowing Screening Test [28], the 3-ounce water swallow 
test [29], and the Eating Assessment Tool-10 [30]. Best practice entails a formal screening for all patients/residents considered to be in at-risk populations (i.e., any neurological condition or head and neck cancer survivor) upon admission if a dysphagia diagnosis has not previously been given, to reduce risk of pneumonia [31] and malnutrition [23] (Chap. 18).

\subsubsection{Other Health Professionals}

In addition to dietitians, physicians, and SLPs, other health professions may be involved in malnutrition prevention and detection. Specifically, occupational therapists may be consulted in acute care to provide strategies and specialized equipment for self-feeding post-paresis or amputation. Together with physical therapists, they may be involved in the assessment of sarcopenia, a condition often comorbid with malnutrition, which also responds to nutritional therapy, as well as physical therapy and exercise (Chaps. 8 and 14). Pharmacists can support optimization of medications, minimizing side effects that impact appetite or food intake (Chap. 20). Social workers support patients with identification of community services such as grocery shopping or help with cooking allowing older adults to leave hospital sooner and remain at home for as long as possible. In acute and long-term care, dietary service aides/nutrition aides provide food to residents and patients and see them on a mealto-meal basis and thus can help to identify preferred foods, especially when intake is poor. All members of the team are essential in encouraging the person to eat, emphasizing the importance of food to their recovery and health, and identifying potential risks with eating that need to be discussed with other specialized members of the team. Allied health providers can also support residents with getting to the dining room and using this time for mobilization.

\subsubsection{The Nursing Role in Malnutrition}

The nursing team links the entire multidisciplinary team together. Consisting of registered nurses, registered or licensed practical nurses, and care aides, nursing staff are essential for all core activities that involve prevention, detection, and treatment of malnutrition (Chaps. 1-6). For example, during their assessment, nurses identify potential challenges that impact food access and consumption. They also complete ongoing monitoring of patients and residents during meals, such as how much is consumed and if eating challenges occur, to not only prevent malnutrition but also determine the effectiveness of treatments. They measure body weight and are commonly the team member that completes nutrition screening and dysphagia screening $[12,32]$. They are integral in identifying potential risk of malnutrition, dysphagia, and/or issues with self-feeding given their consistent interactions with patients and residents. Nurses often initiate referrals to other members of the multidisciplinary team and community services. They can be involved in delivering oral nutritional supplements during medication passes [32], as well as micronutrient 
pills. Where dietitians are unavailable in the community setting, nurses are vital to preventing, detecting, and treating malnutrition of vulnerable older adults and may be involved in providing basic nutrition counseling [33]. As the nursing team is most involved with the patient on a day-to-day basis, it is important that they are well educated on malnutrition (Chap. 12).

\subsubsection{Management and Healthcare Leaders}

Directors of care, managers, and practice and other healthcare leaders also have responsibility for promoting quality nutrition care and prevention of malnutrition in older adults [18]. Specifically, leaders can support a care culture that supports the use of appropriate screening tools, protocols, and standards that are based on best available evidence, quality food; systems that support food delivery, removal of barriers to food intake, and identification and treatment of those who are malnourished; and clarifying roles and training staff on these roles [34].

\subsection{Multidisciplinary Models of Malnutrition Care}

The Making the Most of Mealtimes conceptual model [35] provides a basis for considering the multidisciplinary team approach to promoting food and fluid intake and preventing malnutrition in long-term care. Resident, staff, and home determinants impact food intake, but central to this model is that meal access, meal quality, and mealtime experience directly impact food intake and can be modified by team actions. This conceptual model was confirmed in the Making the Most of Mealtimes prevalence study, identifying that eating assistance, food quality, and mealtime experience were all important for promoting food intake in over 600 residents across 32 long-term care homes [26]. Thus, multidisciplinary care is needed to prevent malnutrition in the residential setting.

Best practice pathways for acute care, transitions, and primary care provide guidance on multidisciplinary care of malnourished patients. Several pathways have been created for specific patient groups. An example is the Integrated Nutrition Pathway for Acute Care [36] designed to provide direction on the prevention, detection, and treatment of malnutrition. Screening is completed on admission by admitting personnel, including nurses and admitting clerks, or potentially by electronic assembly and alerting based on admission data. Those identified to be at risk are assessed, usually by dietitians, using the subjective global assessment [21] to diagnose malnutrition. Standard care practices to promote food intake are commonly identified and implemented by nursing staff, such as reducing barriers to food intake (e.g., the need for eyeglasses and dentures, positioning patients for meals) or determining if the patient is at risk for dysphagia. Monitoring food intake is commonly completed by nursing staff members, such as care aides or dietary aides, to identify improvements and determine if a patient needs intervention before there are significant weight changes [37]. Weight monitoring is also completed. Nurses deliver 
medications and pass out oral nutritional supplements as an advanced care strategy. Dietitians are involved in identifying specialized diet prescriptions for malnourished patients and work with physicians to prescribe enteral or parenteral nutrition as necessary. Discharge planning involves the entire multidisciplinary team to plan for a successful transition home, including referral to the primary care team, continuing dietitian treatment post discharge, and referral to community services. The Primary Care Nutrition Pathway for Hospital to Community Transitions [38] outlines the multidisciplinary roles involved to continue the nutrition care. The primary responsible healthcare provider (e.g., nurse practitioner, family physician) is central to this care, linking malnourished patients to community dietitians and services to support food access and monitoring the nutritional status of patients. When a dietitian is not available, the primary care provider can follow a basic nutrition plan to meet the needs of malnourished patients (https://nutritioncareincanada.ca/resourcesand-tools/primary-community-care/nutrition-care-pathways). The companion Primary Care Nutrition Pathway for Older Adults (65+) provides specific guidance for older adults living in the community and is focused on early detection and prevention. In this pathway, the primary care provider initiates nutrition screening and provides educational resources and a basic nutrition care plan, referring high-risk patients to the dietitian for further assessment and treatment.

\subsection{Standards and Policy Promote Multidisciplinary Malnutrition Care}

In addition to bottom-up approaches by individual healthcare professionals and care institutions to provide quality nutrition care to prevent or treat malnutrition, topdown approaches also improve care. For example, accreditation has been demonstrated to lead to malnutrition screening at a national level [39]. Best practice pathway implementation is supported with policies or standards, as spread and scale are facilitated with the mandate of practices. An example is the Malnutrition Prevention, Detection and Treatment standard from the Health Standards Organization (https://healthstandards.org/), which describes an inclusive and comprehensive approach to multidisciplinary malnutrition care.

\subsection{Examples of Multidisciplinary Malnutrition Care}

The More-2-Eat study [5] is an example of the successful implementation of the multidisciplinary Integrated Nutrition Pathway for Acute Care [36]. Phase 1 demonstrated the feasibility of implementing the pathway in five hospitals and the vital role of the multidisciplinary team. Each hospital was led by a champion who was a nutrition manager, lead dietitian, or nurse. With the support of multidisciplinary site implementation team (nurse, physician, SLP, physiotherapist, food services manager, pharmacist, etc.), the pathway was tailored to the context of each hospital. For example, in one site, physical therapists supported nutrition monitoring by walking 
patients to weigh scales for their biweekly weights [37]. In another site, dietary team members completed admission screening while providing patients with menus and determining their preferences. Food intake monitoring was completed in some sites by the nursing staff, while in one site this was the domain of dietary staff who delivered and picked up trays [37]. Regardless of how the pathway was tailored, success in implementation was demonstrated, including improved practices and patient-reported outcomes and, in four of the five sites, decreased length of stay [32, 40]. Phase 2 demonstrated that implementation could be replicated in more sites and with only the resources available to the hospital [5, 41].

This example highlights the necessity of multidisciplinary nutrition care. There is a role for all members of the healthcare team to prevent, detect, and treat malnutrition with basic care interventions, especially when a dietitian is unavailable [42].

\subsection{Summary}

This chapter has provided an overview of malnutrition, how it is screened and diagnosed, and the role of the multidisciplinary team, across all healthcare sectors, in the prevention, detection, and treatment of malnutrition. Each discipline has a role to play $[42,43]$. Nurses are an integral member of the team, preventing and detecting malnutrition and providing basic nutrition treatment, while linking other team members and community service providers together to ensure that older adults receive the care they need. Conceptual models and best practice pathways for malnutrition guide the process of multidisciplinary care for older adults.

\section{Take-Home Points}

- Malnutrition prevention, detection, and treatment benefit from a multidisciplinary model.

- Pathways of care have been developed to support a multidisciplinary process in malnutrition care for geriatric patients in acute care, transitions, and primary care.

- All health professionals have a role to play, due to the complexity of causes and treatment of geriatric malnutrition.

- Nurses play a central role in coordination of geriatric nutrition care.

\section{References}

1. Cederholm T, Barazzoni R, Austin P, Ballmer P, Biolo G, Compher C et al (2017) ESPEN guidelines on definition and terminology of clinical nutrition. Clin Nutr 36(1):49-64

2. Wolters M, Volkert C, Streicher M, Kiesswetter E, Torbahn G, O'Connor EM et al (2019) Prevalence of malnutrition using harmonized definitions in older adults from different settings-a MaNuEL study. Clin Nutr 38:2389-2398

3. Creda E, Pedrolli C, Klersy C, Bonardi C, Quarleri L, Cappello S et al (2016) Nutritional status in older persons according to healthcare setting: a systematic review and meta-analysis of prevalence data using MNA. Clin Nutr 35:1282-1290 
4. Dent E, Hoogendijk EO, Visvanathan R, Wright ORL (2019) Malnutrition screening and assessment in hospitalised older people: a review. J Nutr Health Aging 23:431-441

5. Keller H, Morrison Koechl J, Laur C, Chen H, Curtis L, Dubin JA et al (2020) More-2-Eat implementation demonstrates that screening, assessment and treatment of malnourished patients can be spread and sustained in acute care; a multi-site, pretest post-test time series study. Clin Nutr 40(4):2100-2108. https://doi.org/10.1016/j.clnu.2020.09.034; S0261-5614(20)30506-9

6. Keller H, Vucea V, Slaughter SE, Jäger-Wittenaar H, Lengyel C, Ottery FD, Carrier N (2019) Prevalence of malnutrition or risk in residents in long term care: comparison of four tools. $\mathrm{J}$ Nutr Gerontol Geriatrics 38(4):329-344. https://doi.org/10.1080/21551197.2019.1640165

7. Marshall S, Craven D, Kelly J, Isenring E (2018) A systematic review and meta-analysis of the criterion validity of nutritional assessment tools for diagnosing protein-energy malnutrition in the older community setting (the MACRo study). Clin Nutr 37:1902-1912

8. Ramage-Morin PL, Garriguet D (2013) Nutritional risk among older Canadians. Health Rep 24(3):3-13

9. Power L, Mullally D, Gibney ER, Clarke M, Visser M, Volkert D et al (2018) A review of the validity of malnutrition screening tools used in older adults in the community and healthcare settings-a MaNuEL study. Clin Nutr 24:1-13

10. Keller HH (2016) Nutri-eSCREEN®: descriptive analysis of a self-management site for older adults (50+ years). BMC Nutr 2:1. https://doi.org/10.1186/s40795-015-0041-7

11. Kaiser MJ, Bauer JM, Ramsch C, Uter W, Guigoz Y, Cederholm T et al (2009) Validation of the Mini Nutritional Assessment short-from (MNA-SF): a practical tool for identification of nutritional status. J Nutr Health Aging 13(9):782-788

12. Laporte M, Keller H, Payette H, Allard JP, Duerksen DR, Bernier P, Jeejeebhoy K, Gramlich L, Vesnaver E, Teterina A (2015) Validity and reliability of the new Canadian Nutrition Screening tool in the 'real-world' hospital setting. Eur J Clin 69(5):558-564. https://doi.org/10.1038/ ejen. 2014.270

13. Keller HH, Goy R, Kane S-L (2005) Validity and reliability of SCREEN II (seniors in the community: risk evaluation for eating and nutrition-version II). Eur J Clin Nutr 59(10):1149-1157

14. Andersen D, Baird S, Bates T, Chapel DL, Cline AD, Ganesh SN et al (2018) Academy of Nutrition and Dietetics: revised 2017 standards of practice in nutrition care and standards of professional performance for registered dietitian nutritionists. J Acad Nutr Diet 118(1):132-140.e15

15. Dorner B, Friedrich EK (2018) Position of the Academy of Nutrition and Dietetics: individualized nutrition approaches for older adults: long-term care, post-acute care and other settings. J Acad Nutr Diet 118:724-735

16. Verlaan S, Ligthart-Melis GC, Wijers SLJ, Cederholm T, Maier AB, de van der Schueren MAE (2017) High prevalence of physical frailty among community-dwelling malnourished older adults-a systematic review and meta-analysis. JAMDA 18:374-382

17. Livingston G, Sommerlad A, Orgeta V, Costafreda SG, Huntley J, Ames D et al (2017) Dementia prevention, intervention, and care. Lancet 390:2673-2734

18. Tappenden KA, Quatrara B, Parkhurst ML, Malone AM, Fanjiang G, Ziegler TR (2013) Critical role of nutrition in improving quality of care: an interdisciplinary call to action to address adult hospital malnutrition. J Acad Nutr Diet 113:1219-1237

19. Fleurke M, Voskuil DW, Beneken Genaamd Kolmer DM (2020) The role of the dietitian in the management of malnutrition in the elderly: a systematic review of current practices. Nutr Diet 77(1):60-75. https://doi.org/10.1111/1747-0080.12546

20. Bell J, Young A, Hill J, Banks M, Comans T, Barnes R, Keller H (2018) Rationale and developmental methodology for The SIMPLE Approach: a Systematized, Interdisciplinary Malnutrition Pathway for implementation and evaluation in hospitals. Nutr Diet 75(2):226-234. https://doi. org/10.1111/1747-0080.12406

21. Detsky AS, McLaughlin JR, Baker JP, Johnston N, Whittaker S, Mendelson RA, Jeejeebhoy KN (1987) What is subjective global assessment of nutritional status? J Parent Enteral Nutr 11(1):8-13 
22. Van den Berg GH, Lindeboom R, van der Zwet WC (2015) The effects of the administration of oral nutritional supplementation with medication rounds on the achievement of nutritional goals: a randomized controlled trail. Clin Nutr 34(1):15-19

23. Namasivayam-MacDonald A, Morrison J, Steele CM, Keller H (2017) How swallow pressures and dysphagia affect malnutrition and mealtime outcomes in long-term care. Dysphagia 32(6):785-796. https://doi.org/10.1007/s00455-017-9925-z

24. Vucea V, Keller HH, Morrison JM, Duncan AM, Duizer LM, Carrier N, Lengyel CO, Slaugther SE (2017) Nutritional quality of regular and pureed menus in Canadian long term care homes: an analysis of the making the most of mealtimes (M3) project. BMC Nutr 3:80. https://doi. org/10.1186/s40795-017-0198-3

25. Takizawa C, Gemmell E, Kenworthy J, Speyer R (2016) A systematic review of the prevalence of oropharyngeal dysphagia in stroke, Parkinson's disease, Alzheimer's disease, head injury, and pneumonia. Dysphagia 31(3):434-441

26. Keller H, Carrier N, Slaughter S, Lengyel C, Steele CM, Duizer L, Morrison J, Brown KS, Chaudhury H, Yoon MN, Duncan AM, Boscart V, Heckman G, Villalon L (2017) Prevalence and determinants of poor food intake or residents living in long term care. J Am Med Dir Assoc 18(11):941-947. https://doi.org/10.1016/j.jamda.2017.05.003

27. Mulheren RW, Azola AM, Kwiatkowski S, Karagiorgos E, Humbert I, Palmer JB, GonzálezFernández M (2018) Swallowing changes in community-dwelling older adults. Dysphagia 33(6):848-856

28. Martino R, Silver F, Teasell R, Bayley M, Nicholson G, Streiner DL, Diamant NE (2009) The Toronto bedside swallowing screening test (TOR-BSST) development and validation of a dysphagia screening tool for patients with stroke. Stroke 40(2):555-561

29. Suiter DM, Leder SB (2008) Clinical utility of the 3-ounce water swallow test. Dysphagia 23(3):244-250

30. Belafsky PC, Mouadeb DA, Rees CJ, Pryor JC, Postma GN, Allen J, Leonard RJ (2008) Validity and reliability of the Eating Assessment Tool (EAT-10). Ann Otol Rhinol Laryngol 117(12):919-924

31. Hinchey JA, Shephard T, Furle K, Smith D, Wang D, Tonn S (2005) Formal dysphagia screening protocols prevent pneumonia. Stroke 36(9):1972-1976

32. Keller H, Valaitis R, Laur CV, McNicholl T, Xu Y, Dubin JA, Curtis L, Obiorah S, Ray S, Bernier P, Gramlich L, Stickles-White M, Laporte M, Bell J (2018) Multi-site implementation of nutrition screening and diagnosis in medical care units: success of the project More-2-Eat. Clin Nutr:1-9. https://doi.org/10.1016/j.clnu.2018.02.009

33. Vasiloglou MF, Fletcher J, Poulia KA (2019) Challenges and perspectives in nutritional counselling and nursing: a narrative review. J Clin Med 8:1489. https://doi.org/10.3390/jcm8091489

34. Keller HH, Vesnaver E, Davidson B, Allard J, Laporte M, Bernier P, Payette H, Jeejeebhoy K, Duerksen D, Gramlich L (2014) Providing quality nutrition care in acute care hospitals: perspectives of nutrition care personnel. J Hum Nutr Diet 27(2):192-202. https://doi.org/10.1111/ jhn. 12170

35. Keller H, Carrier N, Duizer L, Lengyel C, Slaughter S, Steele C (2014) Making the Most of Mealtimes (M3): grounding mealtime interventions with a conceptual model. J Am Med Dir Assoc 15(3):158-161. https://doi.org/10.1016/j.jamda.2013.12.001

36. Keller H, McCullough J, Davidson B, Vesnaver E, Laporte M, Gramlich L, Allard J, Bernier P, Duerksen D, Jeejeebhoy K (2015) Integrated nutrition pathway for acute care (INPAC): building consensus with a modified Delphi. Nutr J 14:63. https://doi.org/10.1186/s12937-015-0051-y

37. Laur C, Nasser R, Butterworth D, Valaitis R, Bell J, Marcell C, Murphy J, Ray S, Bernier P, Keller H (2019) Implementing food intake and body weight monitoring in acute care using behavior change techniques: case examples from the More-2-Eat study. Nutr Clin Pract 34(3):459-474. https://doi.org/10.1002/ncp.10207

38. Keller H, Donnelly R, Laur C, Goharian L, Nasser R (2021) Consensus-based nutrition care pathways for hospital-to-community transitions and older adults in primary and community care. J Paren Enter Nutr (online ahead of print). https://doi.org/10.1002/jpen.2068n 
39. Leistra E, van Bokhorst-de van der Schueren M, Visser M, van der Hout A, Langius J, Kruizenga H (2014) Systematic screening for undernutrition in hospitals: predictive factors for success. Clin Nutr 33(3):495-501

40. Keller H, Xu Y, Dubin JA, Curtis L, Laur C, Bell J, for the More-2-Eat Team (2018) Improving the standard of nutrition care in hospital: mealtime barriers reduced with implementation of the Integrated Nutrition Pathway for Acute Care. Clin Nutr ESPEN 28:74-79

41. Keller H, Koechl JM, Laur C, Chen H, Curtis L, Dubin JA, Gramlich L, Ray S, Valaitis R, Yang Y, Bell J (2021) More-2-Eat implementation demonstrates that screening, assessment and treatment of malnourished patients can be spread and sustained in acute care; a multisite, pretest post-test time series study. Clin Nutr 40(4):2100-2108. https://doi.org/10.1016/j. clnu.2020.09.034

42. Ten Cate D, Ettema RGA, Huisman-de Waal G, Bell JJ, Verbrugge R, Schoonhoven L et al (2020) Interventions to prevent and treat malnutrition in older adults to be carried out by nurses: a systematic review. J Clin Nurs 29(11-12):1883-1902

43. Laur C, Keller H (2015) Implementing best practice in multidisciplinary nutrition care: an example of using the Knowledge-to-Action Process for a research program. J Multidiscip Healthc 8:463-472. https://doi.org/10.2147/JMDH.S93103

\section{Recommended Reading}

Keller H, Carrier N, Duizer L, Lengyel C, Slaughter S, Steele C (2014) Making the Most of Mealtimes (M3): grounding mealtime interventions with a conceptual model. J Am Med Dir Assoc 15(3):158-161. https://doi.org/10.1016/j.jamda.2013.12.001

Keller H, McCullough J, Davidson B, Vesnaver E, Laporte M, Gramlich L, Allard J, Bernier P, Duerksen D, Jeejeebhoy K (2015) Integrated nutrition pathway for acute care (INPAC): building consensus with a modified Delphi. Nutr J 14:63. https://doi.org/10.1186/s12937-015-0051-y

Ten Cate D, Ettema RGA, Huisman-de Waal G, Bell JJ, Verbrugge R, Schoonhoven L et al (2020) Interventions to prevent and treat malnutrition in older adults to be carried out by nurses: a systematic review. J Clin Nurs 29(11-12):1883-1902 
Open Access This chapter is licensed under the terms of the Creative Commons Attribution 4.0 International License (http://creativecommons.org/licenses/by/4.0/), which permits use, sharing, adaptation, distribution and reproduction in any medium or format, as long as you give appropriate credit to the original author(s) and the source, provide a link to the Creative Commons license and indicate if changes were made.

The images or other third party material in this chapter are included in the chapter's Creative Commons license, unless indicated otherwise in a credit line to the material. If material is not included in the chapter's Creative Commons license and your intended use is not permitted by statutory regulation or exceeds the permitted use, you will need to obtain permission directly from the copyright holder.

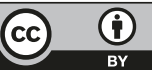

